\title{
RETRACTED ARTICLE: Upregulation of miR-300 and downregulation of miR-125b act as potential predictor biomarkers in progression, metastasis, and poor prognosis of osteosarcoma
}

\author{
Seyyed Hasan Karbasy ${ }^{1}$ - Afshin Taheriazam ${ }^{2}$ - Alireza Mirghasemi ${ }^{3}$. \\ Farnoush Sedaghati ${ }^{4} \cdot$ Mohammadreza Shakeri $^{5} \cdot$ Emad Yahaghi $^{6} \cdot$ Reza Bahador $^{5}$
}

Published online: 2 September 2015

(C) International Society of Oncology and BioMarkers (ISOBM) 2016

\section{RETRACTED ARTICLE: Tumor Biol.}

DOI 10.1007/s13277-015-4000-3

This article has been retracted at the request of the Editorin-Chief, the International Society of Oncology and BioMarkers (ISOBM) and the Publisher per the Committee on Publication Ethics guidelines. The article shows evidence of irregularities in authorship during the submission process, there is strong reason to believe that the peer review process was compromised and there are similarities with the following articles which were all submitted within a close timeframe:

Afshin Taheri Azam \& Reza Bahador \& Hamid Hesarikia \& Mohammadreza Shakeri \& Ali Yeganeh, Down-regulation of

Electronic supplementary material The online version of this article (doi:10.1007/s13277-015-4000-3.) contains supplementary material, which is available to authorized users.

Reza Bahador

rezamdbahador@gmail.com

1 Department of Anesthesiology, Birjand University of Medical Sciences, Birjand, Iran

2 Department of Orthopedics Surgery, Tehran Medical Sciences Branch, Islamic Azad University, Tehran, Iran

3 Department of Orthopedics, Qom University of Medical Sciences, Qom, Iran

4 Pathology Residence, Iran University of Medical Sciences, Tehran, Iran

5 Department of Orthopaedic and Trauma Surgery, Birjand University of Medical Sciences, Birjand, Iran

6 Department of Molecular Biology, Baqiyatallah University of Medical Sciences, Tehran, Iran
microRNA-217 and microRNA-646 acts as potential predictor biomarkers in progression, metastasis and unfavorable prognosis of human osteosarcoma. Tumor Biol. First Online: 31 July 2015 DOI: 10.1007/s13277-015-3821-4

Date received: 5 July 2015

Seyad Alireza Bassampour \& Reza Abdi \& Reza Bahador \& Mohammadreza Shakeri \& Ali Torkaman \& Emad Yahaghi \& Afshin Taheriazam, Downregulation of miR-133b/miR-503 acts as efficient prognostic and diagnostic factors in patients with osteosarcoma and these predictor biomarkers are correlated with overall survival. Tumor Biol. First Online: 16 August 2015 DOI: 10.1007/s13277-015-3918-9

Date received: 20 July 2015

The retracted article was received: 24 July 2015.

As such the validity of the content of this article cannot be verified.

The online version of this article contains the full text of the retracted article as electronic supplementary material. 